\title{
Correction to: Evaluation of two rapid molecular test systems to establish an algorithm for fast identification of bacterial pathogens from positive blood cultures
}

\author{
Philipp Oberhettinger $^{1,2} \cdot$ Jan Zieger ${ }^{1,2} \cdot$ Ingo Autenrieth $^{1,2} \cdot$ Matthias Marschal $^{1,2} \cdot$ Silke Peter $^{1,2}$ \\ Published online: 1 September 2020 \\ (C) Springer-Verlag GmbH Germany, part of Springer Nature 2020
}

\section{Correction to: European Journal of Clinical Microbiology \& Infectious Diseases (2020) 39:1147-1157.} https://doi.org/10.1007/s10096-020-03828-5

Table 2 in the originally published article is not correct and is a duplicate of Table 3 . The error happened during typesetting. The correct Table 2 is shown below.

Table 2 Percentage of detected organisms (\%) on genus and species level, as defined in the system panel for the respective target organism. All isolates are from monomicrobial blood cultures and percentages are shown separately for organisms covered by the respective panel as well as in relation for all microorganisms detected by the reference method (overall)

\begin{tabular}{|c|c|c|c|c|c|c|c|c|c|c|c|}
\hline \multicolumn{4}{|c|}{ Gram-positive $(n=98)$} & \multicolumn{4}{|c|}{ Gram-negative $(n=38)$} & \multicolumn{4}{|l|}{ Yeast $(n=6)$} \\
\hline in panel & & overall & & in panel & & overall & & in panel & & overall & \\
\hline FilmArray® & ePlex® & FilmArray® & ePlex® & FilmArray® & ePlex® & FilmArray® & ePlex® & FilmArray® & ePlex® & FilmArray® & ePlex® \\
\hline 98.9 & 94.7 & 87.8 & 90.8 & 100 & 100 & 81.8 & 87.9 & 100 & 100 & 100 & 100 \\
\hline
\end{tabular}

Publisher's note Springer Nature remains neutral with regard to jurisdictional claims in published maps and institutional affiliations.

The online version of the original article can be found at https://doi.org/ 10.1007/s10096-020-03828-5

Philipp Oberhettinger

philipp.oberhettinger@med.uni-tuebingen.de

1 Institute of Medical Microbiology and Hygiene, University of Tübingen, Elfriede-Aulhorn-Str. 6, 72076 Tübingen, Germany

2 German Center for Infection Research (DZIF), Partner Site Tübingen, Tübingen, Germany 